\title{
Reduced Field-of-View DWI with Robust Fat Suppression and Unrestricted Slice Coverage Using Tilted 2D RF Excitation
}

\author{
Suchandrima Banerjee, ${ }^{1}$ Dwight G. Nishimura, ${ }^{2}$ Ajit Shankaranarayanan, ${ }^{1}$ \\ and Emine Ulku Saritas ${ }^{3,4 *}$
}

Purpose: Reduced field-of-view (rFOV) diffusion-weighted imaging (DWI) using 2D echo-planar radiofrequency (2DRF) excitation has been widely and successfully applied in clinical settings. The purpose of this work is to further improve its clinical utility by overcoming slice coverage limitations without any scan time penalty while providing robust fat suppression.

Theory and Methods: During multislice imaging with 2DRF pulses, periodic sidelobes in the slice direction cause partial saturation, limiting the slice coverage. In this work, a tilting of the excitation plane is proposed to push the sidelobes out of the imaging section while preserving robust fat suppression. The 2DRF pulse is designed using Shinnar-Le Roux algorithm on a rotated excitation k-space. The performance of the method is validated via simulations, phantom experiments, and high in-plane resolution in vivo DWI of the spinal cord.

Results: Results show that rFOV DWI using the tilted 2DRF pulse provides increased signal-to-noise ratio, extended coverage, and robust fat suppression, without any scan time penalty. Conclusion: Using a tilted 2DRF excitation, a high-resolution rFOV DWI method with robust fat suppression and unrestricted slice coverage is presented. This method will be beneficial in clinical applications needing large slice coverage, for example, axial imaging of the spine, prostate, or breast. Magn Reson Med 76:1668-1676, 2016. (c) 2016 International Society for Magnetic Resonance in Medicine

Key words: diffusion-weighted imaging; 2D RF pulse; reduced field-of-view; fat suppression; multislice imaging

${ }^{1}$ Global MR Applications \& Workflow, GE Healthcare, Menlo Park, California, USA.

${ }^{2}$ Department of Electrical Engineering, Stanford University, Stanford, California, USA.

${ }^{3}$ Department of Electrical and Electronics Engineering, Bilkent University, Ankara, Turkey.

${ }^{4}$ National Magnetic Resonance Research Center (UMRAM), Bilkent University, Ankara, Turkey.

Grant sponsor: Supported by the European Commission through an FP7 Marie Curie Career Integration Grant (PCIG13-GA-2013-618834), by the Turkish Academy of Sciences through TUBA-GEBIP 2015 program, and by the BAGEP Award of the Science Academy.

${ }^{*}$ Correspondence to: Emine Ulku Saritas, Department of Electrical and Electronics Engineering, Bilkent University, Ankara, TR-06800, Turkey. E-mail: saritas@ee.bilkent.edu.tr.

Suchandrima Banerjee and Ajit Shankaranarayanan are employed by GE Healthcare.

Received 13 March 2016; revised 11 July 2016; accepted 11 August 2016 DOI 10.1002/mrm.26405

Published online 21 September 2016 in Wiley Online Library (wileyonlinelibrary.com).

(C) 2016 International Society for Magnetic Resonance in Medicine 1668

\section{INTRODUCTION}

Diffusion-weighted imaging (DWI) provides valuable insight into the microstructural organization of tissues and their state of development, disruption, or degeneration (1). Diffusion-weighted imaging is being increasingly included in routine clinical MRI exams (2) because it does not need administration of contrast agents, and because of the relative ease of protocol compared to some other advanced techniques, such as spectroscopic imaging. Over the last few years, two complementary trends have emerged in DWI: whole body DWI-the purpose of which is akin to a screening exam-and reduced field-of-view (rFOV) DWI for targeted imaging. This work focuses on further development of the latter.

The single-shot echo-planar imaging (ssEPI) sequence still remains the workhorse for DWI because of its speed, despite its sensitivity to off-resonance effects. Multishot techniques such as interleaved EPI (3-5) and readoutsegmented EPI (6-9) have been used to reduce offresonance effects by shortening the echo train, but these methods also have challenges arising from motioninduced errors that vary from shot to shot in DWI. An alternative solution is rFOV imaging, which enables zooming into a region of interest while avoiding aliasing artifacts. Obviating the need to encode a large extent in the phase-encode direction shortens the echo train and reduces off-resonance-induced artifacts in ssEPI, resulting in significantly improved image quality. Reduced FOV DWI has been demonstrated using multiple approaches, such as zoonal oblique multislice (ZOOM) EPI (10), outer volume suppression (11) together with partially parallel imaging (12), or two-dimensional (2D) spatially selective excitation $(13,14)$. The $2 \mathrm{D}$ excitation achieves $\mathrm{rFOV}$ by exciting a small phase FOV extent, providing selectivity along both the phase-encode and slice directions.

Previously, we demonstrated rFOV DWI with 2D echoplanar radiofrequency (2DRF) excitation, for which the blipped axis of the echo-planar excitation k-space trajectory was along the slice direction (13). Such a design helps achieve a sharp phase FOV profile that does not require oversampling. Additionally, it provides an inherent fat-suppression capability. This rFOV DWI technique has been successfully applied to several anatomical sites, such as the spine, breast, and prostate (15-21).

One limitation of the rFOV approach using 2DRF pulses is that excitation sidelobes appear along the 

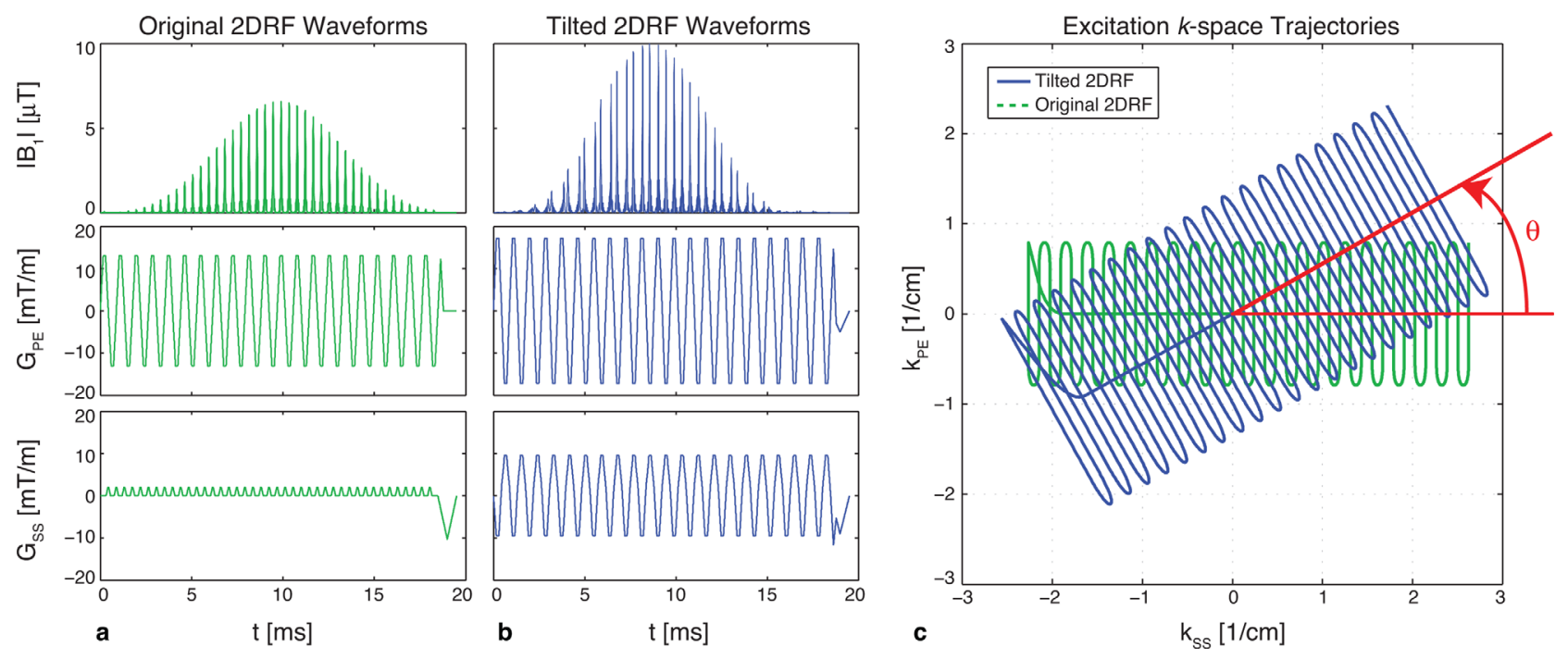

FIG. 1. The RF and gradient waveforms for an example 2D echo-planar RF pulse (a) before and (b) after tilting. (c) The excitation k-space trajectory of the tilted 2DRF pulse is rotated by an angle $\theta$ with respect to the original trajectory. 2D, two dimensional; RF, radiofrequency.

blipped axis of the echo planar trajectory, which can interfere with the imaging section. When the blipped axis is along the slice direction (13), excitation sidelobes can overlap with the imaging section to cause signal loss due to partial saturation, putting a limitation on slice coverage. With a hybrid flyback trajectory, a maximum of eight slices could be imaged when the RF pulse duration is limited to $16 \mathrm{~ms}$. With a blipped trajectory, 16 slices could be imaged for similar RF pulse durations (22). For a generous slice thickness of $5 \mathrm{~mm}, 16$ slices would provide an 8 -cm coverage along the slice direction. However, much larger slice coverage is needed in applications such as axial DWI of the thoracic spine.

An alternative approach for 2DRF pulse design was presented with the blipped axis along the phase-encode direction (14). Although this removed the above-mentioned slice coverage problem, the sharpness of the rFOV profiles was reduced, and the $R F$ pulse had to be quite long ( $\sim 24 \mathrm{~ms})$ to position the sidelobes outside the object in the phaseencode direction. In a follow-up work, a novel way of positioning the sidelobes was proposed: by tilting the 2D excitation plane, the sidelobes were tilted away from the phaseencode direction (23). It should be noted that neither the aforementioned (14) nor the tilted design (23) had inherent fat-suppression capability. Because ssEPI images experience significant shift between fat and water, external fatsuppression schemes were thus required in both cases.

In this work, we propose an analogous tilting approach for the $2 \mathrm{DRF}$ pulse design that we presented in (13): the restriction on slice coverage is eliminated while preserving robust fat-suppression properties. We show with extensive simulations, phantom imaging experiments, and high inplane resolution in vivo axial DWI of the spinal cord that the proposed scheme achieves sharp rFOV profiles without any slice coverage limitations or scan time penalty.

\section{THEORY}

\section{D Echo-Planar RF Pulse Design}

2DRF pulses provide independent control of slab selection and slice selection (SS) during rFOV imaging
$(13,24)$. Here, the slab direction corresponds to the phase-encoding (PE) (or the rFOV) direction during ssEPI readout. In our design approach, the fast direction is the $\mathrm{PE}$ axis, and the slow (i.e., blipped) direction is the SS axis (see Fig. 1a). The 2DRF pulse is given by

$$
B_{1}(t)=C(\alpha) A_{S S}\left(k_{S S}(t)\right) A_{P E}\left(k_{P E}(t)\right)\|G(t)\|
$$

where $C(\alpha)$ sets the flip angle to $\alpha$, and $A_{S S}(k)$ and $A_{P E}(k)$ are the 1D RF pulses designed independently for the SS and $\mathrm{PE}$ directions, respectively. The gradient amplitude $\|G(t)\|$ is utilized to scale the RF power during both the ramps and plateaus of the gradient waveform. Here, $k_{S S}(t)$ and $k_{P E}(t)$ are the SS and PE components of the echo-planar excitation k-space trajectory, that is,

$$
k(t)=k_{S S}(t)+i k_{P E}(t)
$$

With this type of a design, the separation between the periodic lobes in the blipped direction is given by:

$$
\Delta d_{S S}=\frac{N_{\text {blip }}}{T B W_{S S}} \Delta z
$$

where $N_{\text {blip }}$ is the number of blips in the gradient waveform, $T B W_{s s}$ is the time-bandwidth product (TBW) in the slice direction, and $\Delta \mathrm{z}$ is the slice thickness. The upper limit on the number of slices that can be imaged in a single acquisition without inducing any signal loss is determined by the number of slices that can fit between the periodic lobes of the $2 \mathrm{D}$ excitation profile, that is,

$$
N_{\text {max }, \text { slices }}=\frac{\Delta d_{S S}}{\Delta z}=\frac{N_{\text {blip }}}{T B W_{S S}}
$$

To accommodate more slices, one needs to either increase $N_{\text {blip }}$ or reduce $T B W_{s s}$, which would result in a longer RF pulse or reduced sharpness of the slice profile, respectively.

During the echo planar excitation, fat experiences a shift relative to water due to its chemical shift. This 

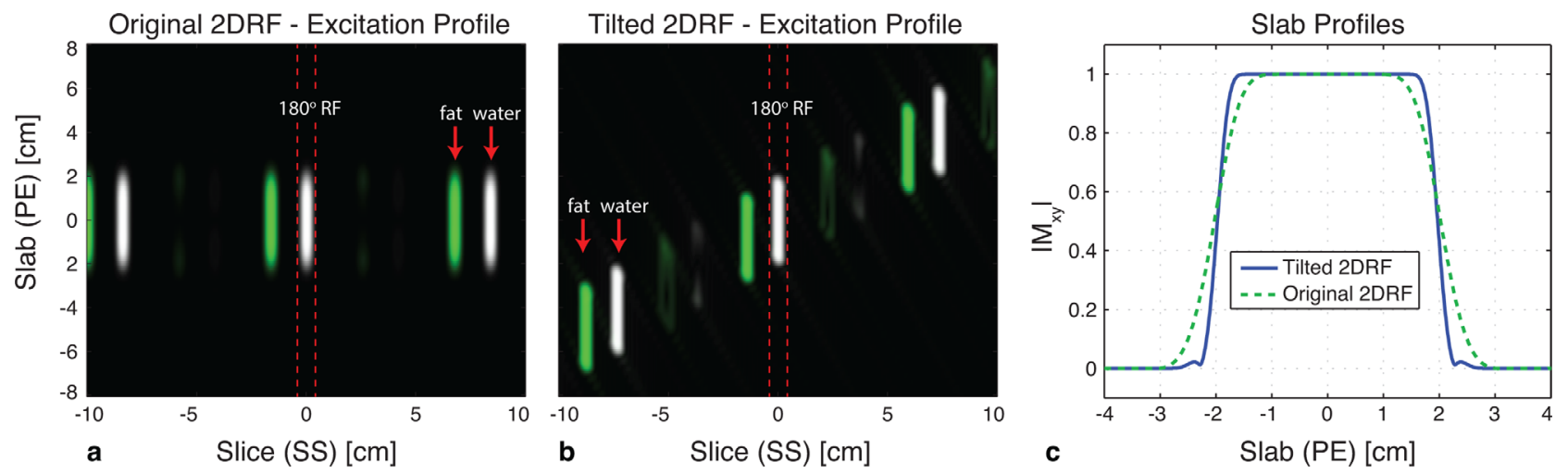

FIG. 2. Simulated 2D excitation profiles for water and fat, for an example 2DRF pulse before and after tilting. For both cases, the 2D profiles are periodic along the blipped direction of the excitation $k$-space trajectory. (a) Because the original 2DRF profile is periodic along the slice direction, the sidelobes cause partial saturation of various slice locations at periodic intervals. (b) The tilted 2DRF profile is periodic along the tilted axis (rotated by $\theta$ with respect to the slice axis). The sidelobes are now pushed outside the potential slice locations, avoiding any potential overlap with the stack of prescribed slices, thus removing restriction in slice coverage. In both designs, the fat profile is shifted with respect to the water profile. Hence, a subsequent $180^{\circ}$ refocusing RF pulse (dashed red lines) selects the mainlobe of the water profile to suppress the signal from fat. (c) The tilted 2DRF design maintains a sharp profile along the slab direction (i.e., the profile along the PE direction during reduced field-of-view imaging). 2D, two-dimensional; 2DRF, two-dimensional radiofrequency; PE, phase encoding; RF, radiofrequency; SS, slice selection.

spatial displacement is along the slice direction and is given by

$$
\Delta d_{C S}=\frac{N_{\text {blip }} f_{C S} T_{\text {fast }}}{T B W_{S S}} \Delta z
$$

where $f_{C S}$ is the chemical shift of fat and $T_{\text {fast }}$ is the duration of one gradient sublobe in the fast direction. In the original design that we presented in (13), $\Delta d_{C S}$ was large enough to ensure no overlap between fat and water excitation profiles, allowing a $180^{\circ}$ refocusing pulse to choose the water profile and suppress the fat signal.

\section{Tilted 2D Echo-Planar RF Pulse Design}

The slice coverage limitation can be removed if the sidelobes are positioned at an angle, outside the imaging section. For this, a $\theta$-degree rotation can be applied to the gradient waveforms, which effectively rotates (or tilts) the echo-planar trajectory, that is,

$$
k_{\text {rot }}(t)=\left(w_{1} k_{S S}(t)+i w_{2} k_{P E}(t)\right) e^{i \theta}
$$

Here, $w_{1}$ and $w_{2}$ are scaling constants to ensure sufficient overlap between the original and rotated excitation kspace trajectories. For the sidelobes to lie completely outside the slice stacks, they need to be pushed up/ down in the $\mathrm{PE}$ direction by a distance equal to or greater than the slab thickness, $\mathrm{FOV}_{\mathrm{PE}}$. The minimum angle of rotation that satisfies this requirement is:

$$
\theta_{\min }=\sin ^{-1}\left(\frac{F O V_{P E}}{N_{\text {max }, \text { slices }} \Delta z}\right)
$$

The 1D RF pulses in SS and PE directions can then be designed on the rotated excitation trajectory using Shinnar-Le Roux transform, with TBWs scaled to preserve the dimensions of the excitation FOV. The tilted 2DRF pulse can be expressed as:

$$
B_{1, \text { rot }}(t)=C(\alpha) A_{S S}\left(k_{S S, \text { rot }}(t)\right) A_{P E}\left(k_{P E, \text { rot }}(t)\right)\left\|G_{\text {rot }}(t)\right\|
$$

where $k_{S S, \text { rot }}(t)=\operatorname{Re}\left\{k_{\text {rot }}(t)\right\}$ and $k_{P E, \text { rot }}(t)=\operatorname{Im}\left\{k_{\text {rot }}(t)\right\}$. An example tilted 2DRF pulse is shown in Figure 1 for $\theta=30^{\circ}, \mathrm{w}_{1}=1$, and $\mathrm{w}_{2}=1.5$; and the resulting $2 \mathrm{D}$ excitation profiles before and after tilting are given in Figure 2. The periodic sidelobes originally positioned along the slice axis (Fig. 2a) appear along the tilted axis after rotation (Fig. 2b), avoiding any potential overlap with the stack of prescribed slices. The 1D slab profile (Fig. 2c) is sharper with the tilted RF pulse because the scaled TBWs were higher than those before tilting. In this example, $\mathrm{FOV}_{\mathrm{PE}}=4 \mathrm{~cm}, \Delta \mathrm{z}=5 \mathrm{~mm}$, and $N_{\text {max,slices }}=16$ before tilting.

It can be shown that, after the tilting process, the separation between water and fat profiles along the tilted axis remains the same as in Eq. [5] for $\mathrm{w}_{1}=1$. The fatwater separation along the actual slice direction can then be calculated as:

$$
\Delta d_{C S, \text { rot }}=\Delta d_{C S} \cos \theta=\frac{N_{\text {blip }} f_{C S} T_{\text {fast }}}{T B W_{S S}} \Delta z \cos \theta
$$

The validity of this equation is verified with the simulations in Figure 2b. As shown in that figure, this slightly reduced fat-water separation still allows for fat suppression using a $180^{\circ}$ refocusing pulse (simulated for $f_{C S}=440 \mathrm{~Hz}$ at $\left.3 \mathrm{~T}\right)$.

\section{METHODS}

\section{DRF Pulses and Simulations}

The 2DRF pulse design and Bloch simulations were implemented in MatLab (MathWorks Inc., Natick, MA). First, the original 2DRF pulse of $16.1 \mathrm{~ms}$ was designed for $\mathrm{FOV}_{\mathrm{PE}}=5 \mathrm{~cm}, \Delta \mathrm{z}=4.5 \mathrm{~mm}, \mathrm{TBW}_{\mathrm{PE}}=8, \mathrm{TBW}_{\mathrm{ss}}=3.1$, and $\mathrm{N}_{\text {max,slices }}=16$. Next, the tilted $2 \mathrm{DRF}$ pulse was designed starting from a similar but slightly longer RF pulse of $19.6 \mathrm{~ms}$ duration and $\mathrm{N}_{\text {max,slices }}=18$, and a 
rotation of $\theta=38^{\circ}$ was applied. The resulting tilted 2DRF pulse also had $19.6 \mathrm{~ms}$ duration. The $2 \mathrm{D}$ excitation profiles for water and fat were simulated before and after tilting, and then they were examined for profile sharpness, distance between periodic lobes, and water-fat separation (simulated for $f_{C S}=440 \mathrm{~Hz}$ at $3 \mathrm{~T}$ ).

Next, we performed Bloch simulations (repetition time $[\mathrm{TR}]=4,400 \mathrm{~ms}$ with an interleaved slice acquisition scheme to match the in vivo MRI experiments) to test the performance and robustness of the tilted 2DRF versus the original 2DRF pulse. Accordingly, the effect of the number of prescribed slices on the signal level was simulated. During Bloch simulations, both the excitation and refocusing RF pulses and accompanying gradients were taken into account, and a typical white matter $\mathrm{T}_{1}$ of $1,100 \mathrm{~ms}$ at $3 \mathrm{~T}$ was assumed. $\mathrm{T}_{2}$ relaxation effects were ignored. The refocusing RF pulse was a standard threelobe sinc-shaped pulse with $3.2 \mathrm{~ms}$ duration and $B W_{r f 180}=905 \mathrm{~Hz}$ bandwidth, exported from the ssEPI DWI sequence used in the MRI experiments.

Both excitation designs were then tested against offresonance sensitivity for frequency offsets between \pm 128 $\mathrm{Hz}$, corresponding to $\pm 1 \mathrm{ppm}$ off-resonance at $3 \mathrm{~T}$. The overall signal loss due to off-resonance was computed by integrating the signal across the slice direction, normalized by the signal level on resonance. These simulations were performed both with and without the refocusing RF pulse included, but ignored $\mathrm{T}_{1} / \mathrm{T}_{2}$ relaxation effects.

\section{MRI Experiments}

MRI experiments were conducted on a GE 3T scanner (MR750, Waukesha, WI) with a head, neck, and spine array coil unit. The ssEPI DWI sequence was programmed such that the conventional 1D excitation pulse could be replaced interchangeably by the two flavors of the 2DRF pulse discussed above.

First, the fat-suppression capability of the tilted 2DRF pulse was experimentally verified by imaging an oilwater phantom. Next, two back-to-back acquisitions were acquired with each excitation technique in a Sphere Phantom (GE, Waukesha, WI) doped with CuSO4 at room temperature for the purposes of signal-to-noise ratio (SNR) measurements (TR/echo time (TE) $=4,400 / 75$ ms, readout bandwidth $(\mathrm{RBW})= \pm 167 \mathrm{kHz}$, number of excitations $(\mathrm{NEX})=4$, acquisition matrix $=128 \times 64$, $62.5 \%$ partial k-space coverage in PE direction, $\mathrm{FOV}=10$ $\times 5 \mathrm{~cm}^{2}, 28$ slices, slice thickness/gap $\left.=4.8 / 5 \mathrm{~mm}\right)$. Although the original 2DRF pulse was designed for maximum slice coverage of 16 slices, we prescribed 28 slices for both flavors of the excitation pulse to investigate SNR loss from partial saturation. Signal-to-noise ratio was calculated using the mean signal from the sum of the two acquisitions and the standard deviation of noise from the difference image (25).

For in vivo imaging experiments, four human subjects were scanned in accordance with the institutional review board protocol. In the first subject, high in-plane resolution axial DWI images of the entire thoracic spine were acquired with the tilted 2DRF pulse (36 slices). The same parameters were then prescribed using the original $2 \mathrm{DRF}$ to investigate SNR loss from partial saturation. In

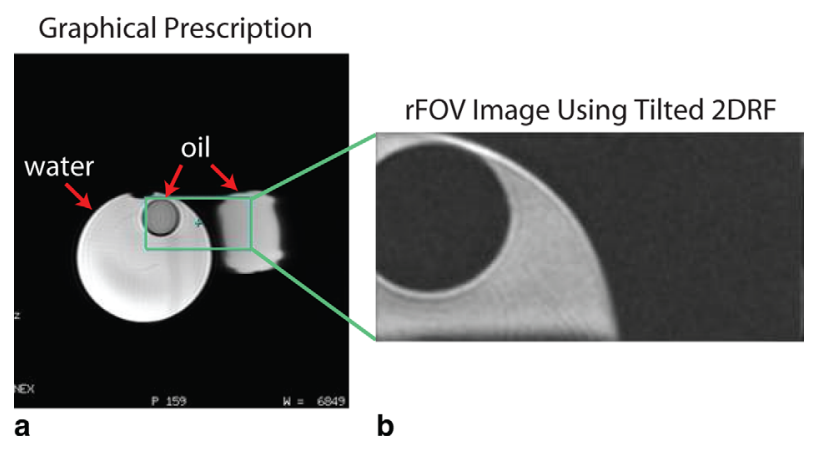

FIG. 3. Experimental verification of the fat-suppression capability of the tilted 2DRF pulse design. (a) A localizer image showing the oil-water phantom setup (with one oil phantom placed in a small tube inside the water phantom, and another oil phantom on the right). The rFOV graphical prescription is shown with the green box. (b) The corresponding $\mathrm{T}_{2}$-weighted rFOV ssEPI image. The grayscale window of this image is adjusted to show that the oil phantoms are completely invisible, demonstrating the reliable fat suppression of the tilted 2DRF pulse. 2DRF, two-dimensional radiofrequency; rFOV, reduced field-of-view ssEPI, single-shot echo-planar imaging.

the next three subjects, axial DWI scans of the thoracic spine or cervical spine were acquired. With the tilted 2DRF pulse, the number of slices prescribed was dictated by the anatomy (32-40 slices). With the original 2DRF pulse, the number of slices was set to the design limit of 16 slices. All remaining scan parameters were kept identical for all in vivo scans $\left(\mathrm{FOV}=10 \times 5 \mathrm{~cm}^{2}, 0.78 \times\right.$ $0.78 \mathrm{~mm}^{2}$ in-plane resolution, slice thickness/gap $=4.8$ / $0 \mathrm{~mm}, \quad \mathrm{TR} / \mathrm{TE}=4,400 / 65 \mathrm{~ms}, \mathrm{RBW}= \pm 167 \mathrm{kHz}$, $\mathrm{NEX}=12,62.5 \%$ partial k-space coverage in PE direction, acquisition matrix $=128 \times 64, b=500 \mathrm{~s} / \mathrm{mm}^{2}$, diffusion in three orthogonal directions). The relatively low $b$ value of $500 \mathrm{~s} / \mathrm{mm}^{2}$ was chosen to ensure sufficient SNR despite the high in-plane resolution (26).

\section{RESULTS}

A 3D localizer image showing the oil-water phantom setup and the rFOV graphical prescription is shown in Figure $3 \mathrm{a}$, and the corresponding $\mathrm{T}_{2}$-weighted rFOV ssEPI image is shown in Figure 3b. Even though the prescribed FOV includes both water and oil, the oil is completely invisible in the rFOV image, demonstrating reliable fatsuppression capability of the tilted 2DRF pulse.

Figure 4 shows simulation results for the effects of the number of prescribed slices on signal level. The mean signal levels across all slices are plotted, with the error bars denoting the minimum and maximum signal levels. Both the tilted and the original 2DRF pulses experience a negligible $4 \%$ signal loss when the number of slices is increased from one to four. For the original 2DRF pulse, once the limit of $\mathrm{N}_{\text {max,slices }} \approx 16$ is exceeded, the sidelobe locations previously excited experience partial saturation effects; as a result, the signal level goes down. Because $\mathrm{N}_{\text {max,slices }}$ is not exactly integer-valued, the signal loss is not the same at all slice locations (i.e., some slices experience partial saturation due to partial overlap of the sidelobes). The mean signal level monotonically goes down as the number of prescribed slices increases, 


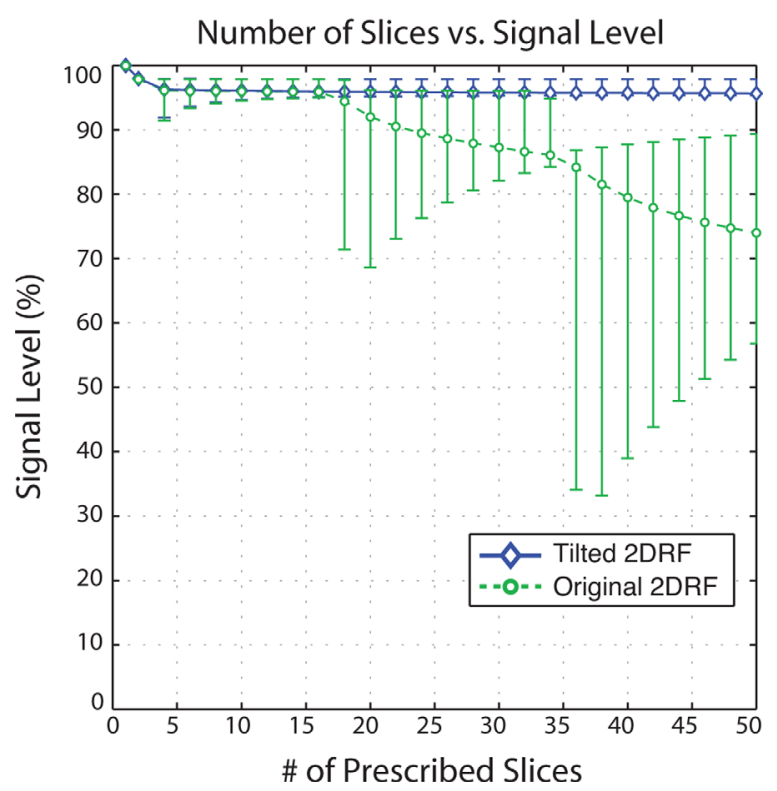

FIG. 4. Simulations results for the effects of the number of prescribed slices on signal level. The mean signal levels across all slices are plotted, with the error bars denoting the minimum and maximum signal levels. Once the maximum slice design limit is exceeded for the original 2DRF (16 slices in this case), it experiences inconsistent signal loss across slice locations. The tilted 2DRF design is immune to such effects. These Bloch simulations were performed including the refocusing RF pulse, with an interleaved slice acquisition scheme for repetition time $=4,400 \mathrm{~ms}$ and $\mathrm{T}_{1}=1,100 \mathrm{~ms}$. 2DRF, twodimensional radiofrequency; $\mathrm{RF}$, radiofrequency.

with $74 \%$ signal level at $\mathrm{N}_{\text {slices }}=50$. Note that although the mean signal shows this monotonic trend, the worstcase scenario (i.e., the minimum signal level among the slices) displays a more erratic behavior. Upon closer inspection, there are local minima around integer multiples of $\mathrm{N}_{\text {max,slices }}$ because this causes the sidelobes to overlap with higher probability with the imaged slice locations. For example, the minimum signal level is at $95 \%$ for $\mathrm{N}_{\text {slices }}=16$ and falls down to $71 \%$ for $\mathrm{N}_{\text {slices }}=18$; it then gradually rises to $84 \%$ for $\mathrm{N}_{\text {slices }}=34$ and falls down to $34 \%$ for $\mathrm{N}_{\text {slices }}=36$. In sharp contrast, the tilted 2DRF pulse is immune to this problem because it displays constant signal levels independent of the number of prescribed slices.

In the SNR experiment in the sphere phantom, 28 slices were prescribed and the SNR of each slice for the original versus the tilted 2DRF cases was measured (images not shown). No significant difference in SNRs was found between the two cases for the first group of 16 slices (paired t-test, with $P>0.09$ ). On the other hand, SNR was significantly higher (paired t-test, with $P<0.002$ ) with the tilted $2 \mathrm{DRF}$ pulse for the remaining group of 12 slices. This result is in agreement with the simulation results in Figure 4.

Figures 5a and 5b show in vivo axial DWI images from the top and bottom of the slice stack and sagittal reformats in the thoracic spine. For this comparison, slice coverage of 36 slices was matched between the tilted and original 2DRF acquisitions, exceeding the 16-slice design limit of the original 2DRF pulse. As seen in these images, the inferior part of the spine has similar SNR between the two cases. SNR is lower for both cases in the superior part of the spine where the spinal cord curves away from the receiver array. However, the SNR deterioration is much more drastic for the image from the original 2DRF case due to aforementioned partial saturation effects, displaying the increased SNR advantage of the tilted 2DRF excitation.

Figures 5c and 5d demonstrate the case when 16 slices were acquired with the original $2 \mathrm{DRF}$ pulse (i.e., at its design limit), whereas 38 slices were acquired for the tilted 2DRF case. These results show that the tilted 2DRF pulse provides extended coverage without compromising image quality and without any increase in scan time. Similar results were seen for the other two in vivo scans (results not shown), for which the original 2DRF excitation was used to acquire 16 slices, and the tilted 2DRF excitation was used to acquire as many slices as required by the anatomy (32-40 slices).

Shown in Figure 6 is the off-resonance robustness of the tilted versus original 2DRF pulses, considering the excitation response only. Figures $6 \mathrm{a}$ through $\mathrm{b}$ shows the mainlobes of the 2D excitation profiles for $\Delta \mathrm{f}=0 \mathrm{~Hz}$ (i.e., on-resonance profile) and $\Delta \mathrm{f}=64 \mathrm{~Hz}$ for both versions of the 2DRF pulse. Due to their long durations, the excitation profiles of 2DRF pulses experience a spatial shift along the blipped gradient direction. As a direct result of this effect, the regular 2DRF profile shifts along the slice direction, whereas the tilted 2DRF profile shifts along the tilted axis (i.e., simultaneous shift along the slice and slab directions). Figures $6 \mathrm{c}$ and $6 \mathrm{~d}$ show the dependence of these shifts on resonant offsets. The tilted 2DRF pulse displays slightly reduced shift along the slice direction when compared to the original 2DRF pulse (e.g., $1.7 \mathrm{~mm}$ vs. $1.9 \mathrm{~mm}$ shift at $\Delta \mathrm{f}=64 \mathrm{~Hz}$ ), while displaying a small shift along the slab direction (e.g., $1.3 \mathrm{~mm}$ at $\Delta \mathrm{f}=64 \mathrm{~Hz}$ ). Note that the shift along the slab direction can mostly be ignored when compared to the $50-\mathrm{mm}$ size of the entire slab.

When compared to the 2DRF pulses, the $180^{\circ} \mathrm{RF}$ pulse leads to a smaller spatial shift along the slice direction for a given resonance offset, which can be expressed as $\Delta z \cdot \Delta f / B W_{r f 180}$. Due to the differences in the spatial shifts of the $2 \mathrm{DRF}$ and $180^{\circ} \mathrm{RF}$ pulses, the excited slice can only be refocused partially, as demonstrated in Figure $7 \mathrm{a}$ for $\Delta \mathrm{f}=64 \mathrm{~Hz}$. When compared to the case of $\Delta \mathrm{f}=0 \mathrm{~Hz}$ (given as a reference), the effective slice profile at $\Delta \mathrm{f}=64 \mathrm{~Hz}$ is slightly off-centered and has a reduced width. Next, the effective slice center is computed as the location maxima of the effective slice profile and plotted in Figure $7 \mathrm{~b}$ as a function of resonance offset. The overall signal loss due to off-resonance is computed by integrating over the effective slice profile, normalized by the on-resonant signal level. The result is given in Figure 7c: the signal levels for the tilted 2DRF and original 2DRF cases are at $86 \%$ versus $83 \%$ at $\Delta \mathrm{f}=64 \mathrm{~Hz}$. Note that the spatial shifts of both the excitation profile (see Eq. [9]) and the refocusing profile are proportional to the slice thickness. Hence, for a given $2 \mathrm{DRF} / 180^{\circ} \mathrm{RF}$ pulse pair, the percentage signal loss due to partial refocusing is independent of the prescribed slice thickness. Therefore, we deduce that the tilted 2DRF pulse performs slightly 
Improved SNR

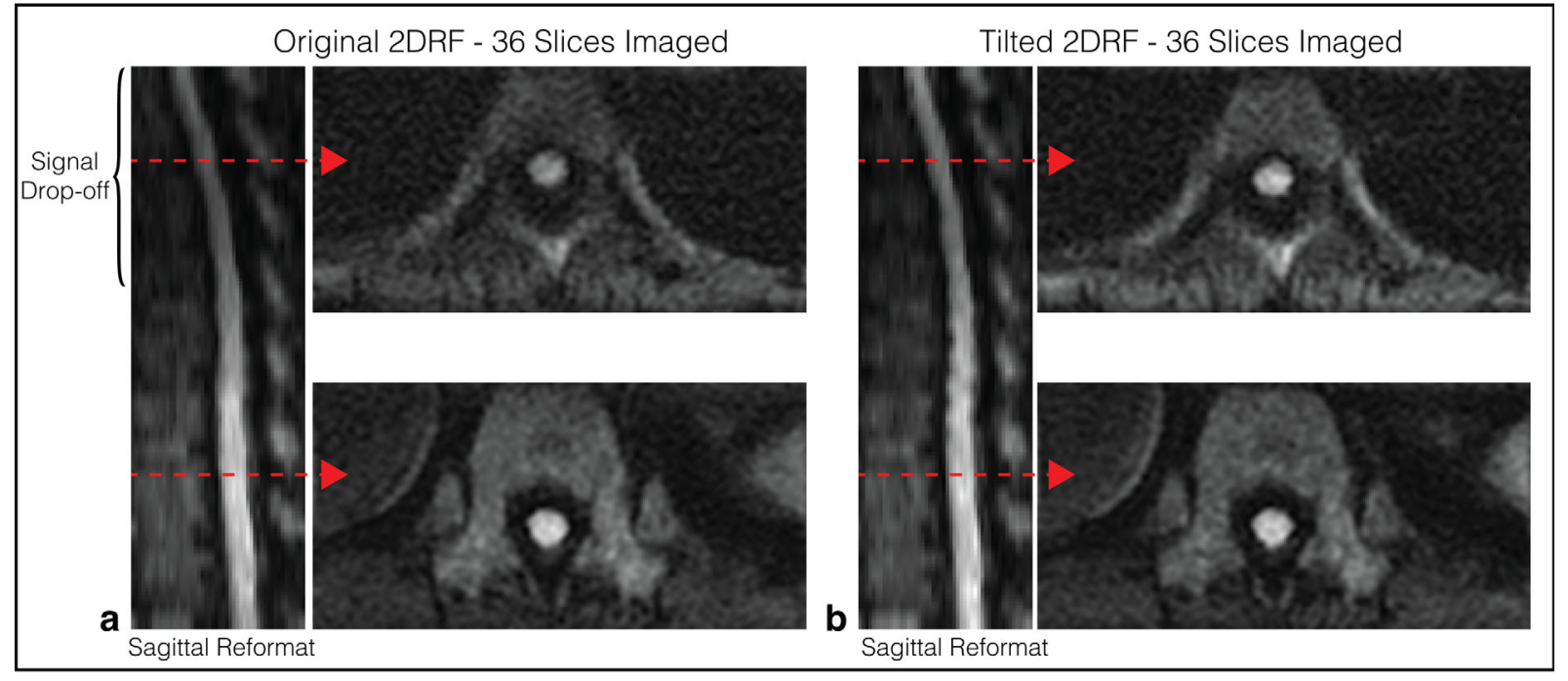

Extended Coverage

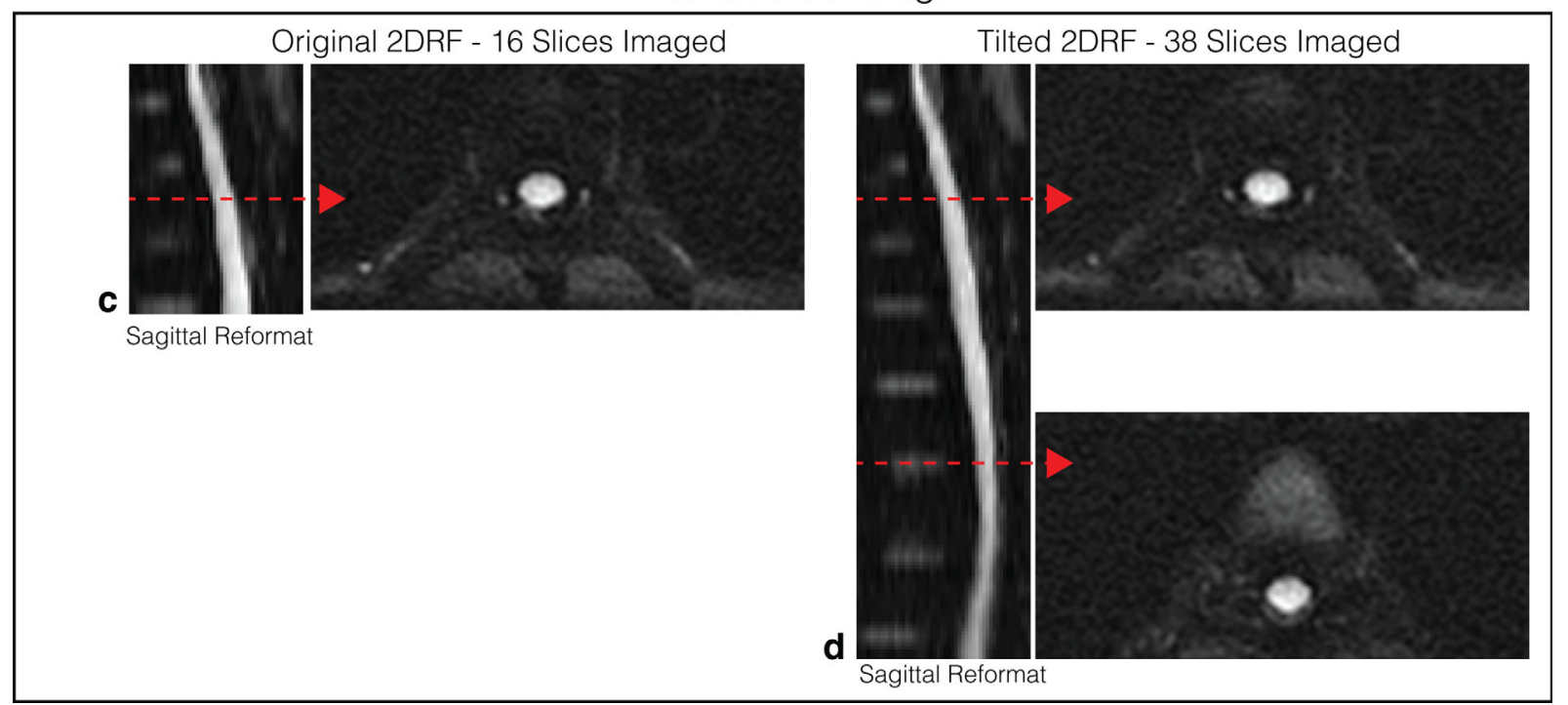

FIG. 5. In vivo results from high-resolution axial DWI of the thoracic spinal cord from two different subjects. (a-b) 36 slices were acquired using the original 2DRF and tilted 2DRF, exceeding the 16-slice design limit of the original 2DRF pulse. SNR is lower for both cases at the top of the stack where the spinal cord curves away from the receiver array. However, the SNR dropoff is much more drastic in the image from the original 2DRF, displaying the SNR advantage of the tilted 2DRF pulse design. (c-d) 16 slices and 38 slices were acquired using the original and the tilted 2DRF pulses, respectively. The tilted 2DRF pulse provides extended coverage without compromising image quality and without any increase in scan time. Field-of-view $=10 \times 5 \mathrm{~cm}^{2}, 0.78 \times 0.78 \mathrm{~mm}^{2}$ in-plane resolution, slice thickness $=4.8 \mathrm{~mm}$ for all axial images. 2DRF, two-dimensional radiofrequency; DWI, diffusion-weighted imaging; SNR, signal-tonoise ratio.

better than the original 2DRF pulse in terms of offresonance robustness.

\section{DISCUSSION}

This work demonstrated a tilted 2DRF excitation pulse, which removed the restriction on slice coverage arising from signal saturation considerations while maintaining robust fat suppression in rFOV ssEPI DWI. Through simulations and experiments, it was shown that prescribing more slices than the design limit of the original 2DRF pulse can lead to considerable and inconsistent signal loss across slices, which in turn might compromise image quality. This problem can also potentially impact quantification of DWI metrics grouped or averaged over slices. Images acquired with the tilted 2DRF are immune to such issues. The limitation in slice coverage in the tilted 2DRF pulse case is determined only by considerations such as coil coverage and the number of slice excitations that can be accommodated within a TR. Through simulations and experiments, it was also demonstrated that the tilted 2DRF design provides inherent and robust fat suppression, as in the original design. Fatsuppression capability is an important consideration in 


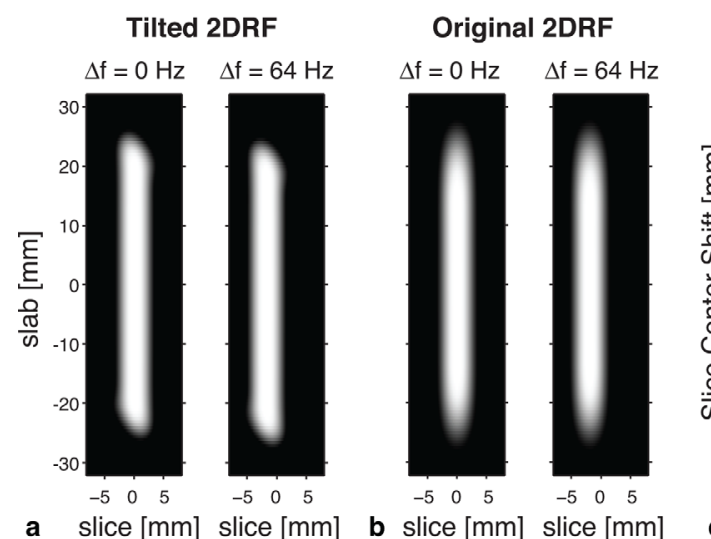

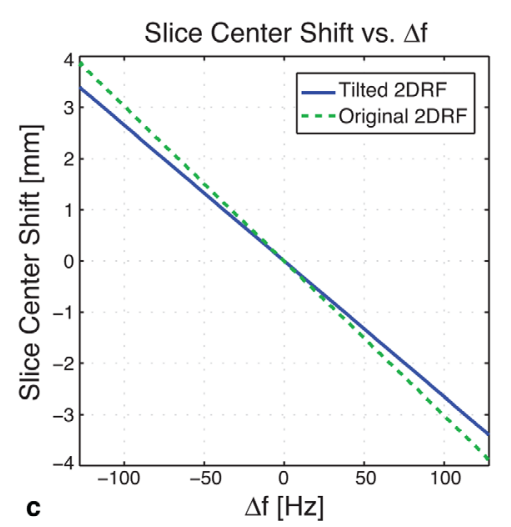

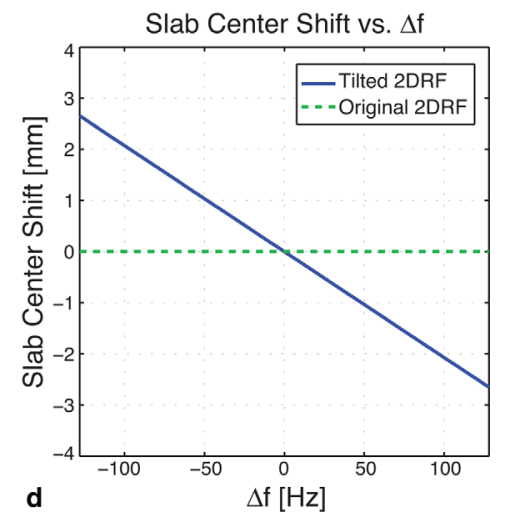

FIG. 6. Simulation results for robustness of the $2 \mathrm{D}$ excitation profile against off-resonance. (a-b) The mainlobes of the $2 \mathrm{D}$ excitation profiles for $\Delta f=0 \mathrm{~Hz}$ (i.e., on-resonance profile) and $\Delta f=64 \mathrm{~Hz}$. Due to their long durations, the excitation profiles experience a spatial shift along the blipped gradient direction in the case of off-resonance. (c-d) The original 2DRF profile shifts along the slice direction only, whereas tilted 2DRF profile shifts along the tilted axis (i.e., simultaneous shift along the slice and slab directions). This effect has a linear dependence on frequency offset. 2D, two-dimensional; 2DRF, two-dimensional radiofrequency.

DWI; chemical shift causes the fat image to be displaced by a few $\mathrm{cm}$ along the phase FOV direction in ssEPI images. For the rFOV imaging case, because the phase FOV is only a few $\mathrm{cm}$, the fat image experiences aliasing, causing image quality failures. The tilted 2DRF pulse prevents these problems by completely suppressing the signal from fat.

Although the RF amplitude increases after the tilting process, the resulting $10 \mu \mathrm{T}$ peak $\mathrm{B}_{1}$ is lower than the typical $15 \mu \mathrm{T}$ hardware limit. The increase in $\mathrm{B}_{1}$ also causes higher specific absorption rates (SAR). For the case shown in Figure 1, tilting caused a $65 \%$ increase in RF power deposition. Nonetheless, this tilted 2DRF pulse still had $3 \%$ lower power deposition than a regular $90^{\circ}$ excitation pulse (sinc-shaped pulse, $3 \mathrm{~ms}$ duration, $\mathrm{TBW}=4,8 \mu \mathrm{T}$ peak $\mathrm{B}_{1}$; compared via time integral of $\left.\left|B_{1}\right|^{2}\right)$. This result stems from the slowed-down nature of the 2DRF pulses, where an effective $90^{\circ}$ excitation stretches over 15 to $20 \mathrm{~ms}$ durations. Hence, the proposed DWI sequence is comfortably within the SAR safety limits for multislice imaging with extended slice coverage. The tilting also caused an increase in gradient amplitudes, mostly due to the usage of $\mathrm{w}_{2}>1$. Still, the tilted gradient waveforms were well below the typical 40 $\mathrm{mT} / \mathrm{m}$ hardware limit. In this study, to ensure that the slew rate limits $\left(\mathrm{SR}_{\max }\right)$ are not exceeded, the gradient waveforms before tilting, were designed using a lower slew rate that satisfied $\mathrm{SR}_{\max }<\mathrm{SR}_{\max } / \mathrm{w}_{2}$.

One potential limitation of the technique is that there may not be a $\theta$ that satisfies Eq. [7] for relatively small slice thicknesses. In such cases, the proposed approach can be modified to design an effective tilted 2DRF. For example, a tilted RF pulse for a thicker slice that yields a solution for $\theta$ can first be designed. The SS gradients could then be scaled upward to shrink the resulting 2D excitation profile to yield a thinner slice. For these cases, hardware limits on maximum gradient strength may need to be carefully considered in the design. Demonstrating the effectiveness of this alternative solution remains a future work.
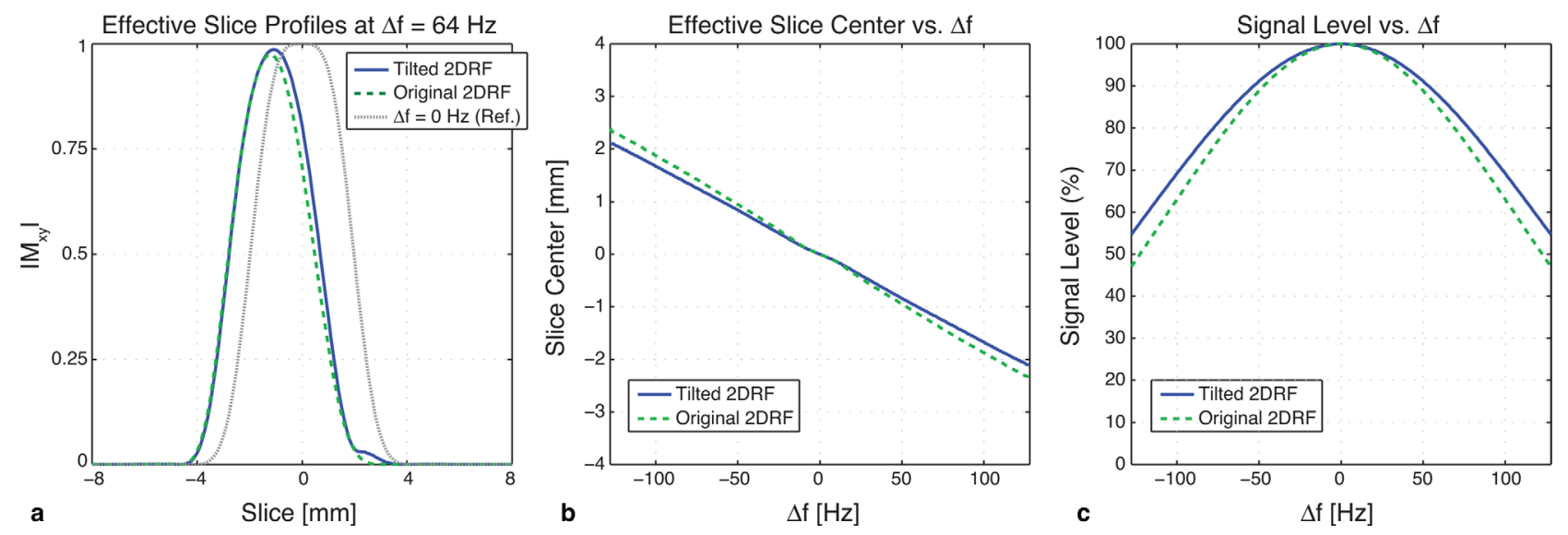

FIG. 7. Overall effect of off-resonance on signal level, considering both the excitation and the refocusing profiles. (a) The effective slice profiles after applying the 2DRF and $180^{\circ} \mathrm{RF}$ pulses at $64 \mathrm{~Hz}$ off-resonance (the effective profile for $\Delta \mathrm{f}=0 \mathrm{~Hz}$ case given as a reference). (b) Effective slice center shift as a function of off-resonance, and (c) The overall signal level is computed by integrating over the effective slice profile, normalized by the on-resonant signal level. The signal levels for the tilted 2DRF and original 2DRF cases are at $86 \%$ vs. $83 \%$ at $\Delta f=64 \mathrm{~Hz}$. 2DRF, two-dimensional radiofrequency; $R F$, radiofrequency. 
As shown in Figures 6 and 7, the tilted 2DRF pulse displays slightly improved off-resonance robustness when compared to the original 2DRF pulse. This result cannot be generalized, however, because the comparison depends heavily on the exact parameters of the two pulses. More generally, we can claim that the tilted 2DRF pulse is comparable to the original 2DRF pulse in terms of off-resonance sensitivity.

It should be noted that the fat-suppression capability of the proposed method is a direct result of the offresonance-induced signal loss; whereas $64 \mathrm{~Hz}$ offresonance causes the signal to drop to $86 \%$ (see Fig. 7), $440 \mathrm{~Hz}$ chemical shift of fat results in a complete loss of signal. As mentioned in the Introduction, the tilted 2DRF scheme presented in (23) did not feature a fatsuppression capability. Consequently, that scheme has the advantage of being more robust against off-resonance effects.

Previously, we have proposed alternative approaches for extending the slice coverage of the original 2DRF design by using a matched multiband refocusing pulse to refocus multiple excitation sidelobes simultaneously, with Hadamard slice encoding (20) or parallel imaging (27) to resolve the simultaneously excited and refocused locations. Each method has its own benefits and pitfalls. The Hadamard-encoding method necessitates multiple averages and can be prone to motion-induced phase errors. The parallel imaging method uses coil-sensitivity information to resolve the simultaneously acquired slices (28-31), for which the reconstruction quality heavily depends on acceleration capabilities of the coil in the slice direction. In contrast, the current work proposes a modification of the excitation pulse itself and does not require multiple averages, specialized reconstruction, or coil with acceleration capabilities in the slice direction.

\section{CONCLUSION}

In this work, we have proposed a scheme of tilting the excitation plane to eliminate restrictions in slice coverage of a 2DRF pulse design for rFOV DWI. This extended slice coverage is achieved without any scan time penalty. In contrast to previously presented tilted 2DRF design, our approach provides a robust fat-suppression capability. The feasibility of using this technique for high inplane resolution rFOV DWI with large slice coverage has been shown for in vivo axial DWI of the spinal cord. Any rFOV DWI application requiring large slice coverage, such as axial imaging of the prostate, breast, or peripheral nerves, will also benefit from the proposed technique.

\section{ACKNOWLEDGMENT}

Parts of this work were presented in the Annual Meeting of the International Society for MR in Medicine in Salt Lake City, Utah, USA, 20-26 April 2013.

\section{REFERENCES}

1. Reeder SB, Mukherjee P. Clinical applications of MR diffusion and perfusion imaging: preface. Magn Reson Imaging Clin N Am 2009;17: xi-xii.
2. Chilla GS, Tan CH, Xu C, Poh CL. Diffusion weighted magnetic resonance imaging and its recent trend-a survey. Quant Imaging Med Surg 2015;5:407-422.

3. Guhaniyogi S, Chu ML, Chang HC, Song AW, Chen NK. Motion immune diffusion imaging using augmented MUSE for highresolution multi-shot EPI. Magn Reson Med 2016;75:639-652.

4. Hayes LL, Jones RA, Palasis S, Aguilera D, Porter DA. Drop metastases to the pediatric spine revealed with diffusion-weighted MR imaging. Pediatr Radiol 2012;42:1009-1013.

5. Skare S, Newbould RD, Clayton DB, Albers GW, Nagle S, Bammer R. Clinical multishot DW-EPI through parallel imaging with considerations of susceptibility, motion, and noise. Magn Reson Med 2007;57: 881-890.

6. Robson MD, Anderson AW, Gore JC. Diffusion-weighted multiple shot echo planar imaging of humans without navigation. Magn Reson Med 1997;38:82-88.

7. Porter DA, Mueller E. Multi-shot diffusion-weighted EPI with readout mosaic segmentation and 2D navigator correction. In Proceedings of the 12th Annual Meeting of ISMRM, Kyoto, Japan, 2004. p. 442.

8. Porter DA, Heidemann RM. High resolution diffusion-weighted imaging using readout-segmented echo-planar imaging, parallel imaging and a two-dimensional navigator-based reacquisition. Magn Reson Med 2009;62:468-475.

9. Holdsworth SJ, Skare S, Newbould RD, Guzmann R, Blevins NH, Bammer R. Readout-segmented EPI for rapid high resolution diffusion imaging at 3 T. Eur J Radiol 2008;65:36-46.

10. Wheeler-Kingshott CA, Parker GJ, Symms MR, Hickman SJ, Tofts PS, Miller DH, Barker GJ. ADC mapping of the human optic nerve: increased resolution, coverage, and reliability with CSF-suppressed ZOOM-EPI. Magn Reson Med 2002;47:24-31.

11. Wilm BJ, Svensson J, Henning A, Pruessmann KP, Boesiger P, Kollias SS. Reduced field-of-view MRI using outer volume suppression for spinal cord diffusion imaging. Magn Reson Med 2007;57:625-630.

12. Heidemann RM, Anwander A, Feiweier T, Knösche TR, Turner R. Turner. k-space and q-space: combining ultra-high spatial and angular resolution in diffusion imaging using ZOOPPA at 7 T. Neuroimage 2012;60:967-978.

13. Saritas EU, Cunningham CH, Lee JH, Han ET, Nishimura DG. DWI of the spinal cord with reduced FOV single-shot EPI. Magn Reson Med 2008;60:468-473.

14. Finsterbusch J. High-resolution diffusion tensor imaging with inner field-of-view EPI. J Magn Reson Imaging 2009;29:987-993.

15. Zaharchuk G, Saritas EU, Andre JB, Chin CT, Rosenberg J, Brosnan TJ, Shankaranarayan A, Nishimura DG, Fischbein NJ. Reduced fieldof-view diffusion imaging of the human spinal cord: comparison with conventional single-shot echo-planar imaging. AJNR Am J Neuroradiol 2011;32:813-820.

16. Andre JB, Zaharchuk G, Saritas E, Komakula S, Shankaranarayan A, Banerjee S, Rosenberg J, Nishimura DG, Fischbein NJ. Clinical evaluation of reduced field-of-view diffusion-weighted imaging of the cervical and thoracic spine and spinal cord. AJNR Am J Neuroradiol 2012;33:1860-1866.

17. Singer L, Wilmes LJ, Saritas EU, Shankaranarayanan A, Proctor E, Wisner DJ, Chang B, Joe BN, Nishimura DG, Hylton NM. High-resolution diffusion-weighted magnetic resonance imaging in patients with locally advanced breast cancer. Acad Radiol 2012;19:526-534.

18. Wilmes LJ, McLaughlin RL, Newitt DC, et al. High-resolution diffusionweighted imaging for monitoring breast cancer treatment response. Acad Radiol 2013;20:581-589.

19. Karampinos DC, Melkus G, Shepherd TM, Banerjee S, Saritas EU, Shankaranarayanan A, Hess CP, Link TM, Dillon WP, Majumdar S. Diffusion tensor imaging and T2 relaxometry of bilateral lumbar nerve roots: feasibility of in-plane imaging. NMR Biomed 2013;26: 630-637.

20. Saritas EU, Lee D, Çukur T, Shankaranarayanan A, Nishimura DG. Hadamard slice encoding for reduced-FOV diffusion-weighted imaging. Magn Reson Med 2014;72:1277-1290.

21. Korn N, Kurhanewicz J, Banerjee S, Starobinets O, Saritas E, Noworolski S. Reduced-FOV excitation decreases susceptibility artifact in diffusion-weighted MRI with endorectal coil for prostate cancer detection. Magn Reson Imaging 2015;33:56-62.

22. Saritas EU, Shankaranarayan A, Zaharchuk G, Nishimura DG. Reduced-FOV single-shot diffusion-weighted EPI: extended slice coverage with tailored RF pulse design. In Proceedings of the 19th Annual Meeting of ISMRM, Montréal, Canada, 2011. p. 1953. 
23. Finsterbusch J. Improving the performance of diffusion-weighted inner field-of-view echo-planar imaging based on 2D-selective radiofrequency excitations by tilting the excitation plane. J Magn Reson Imaging 2012;35:984-992.

24. Alley MT, Pauly JM, Sommer FG, Pelc NJ Angiographic imaging with 2D RF pulses. Magn Reson Med 1997;37:260-267.

25. Reeder SB, Wintersperger BJ, Dietrich O, Lanz T, Greiser A, Reiser MF, Glazer GM, Schoenberg SO. Practical approaches to the evaluation of signal-to-noise ratio performance with parallel imaging: application with cardiac imaging and a 32-channel cardiac coil. Magn Reson Med 2005;54:748-754.

26. Saritas EU, Lee JH, Nishimura DG. SNR dependence of optimal parameters for apparent diffusion coefficient measurements. IEEE Trans Med Imaging 2011;30:424-437.

27. Banerjee S, Saritas EU, Connett R, Shankaranarayanan A. Reduced field-of-view diffusion with 2D echo-planar RF excitation and Multiband refocusing for extended slice coverage and robust fat suppression. In Proceedings of the 22nd Annual Meeting of ISMRM, Milan, Italy, 2014. p. 4437.

28. Breuer FA, Blaimer M, Heidemann RM, Mueller MF, Griswold MA, Jakob PM. Controlled aliasing in parallel imaging results in higher acceleration (CAIPIRINHA) for multi-slice imaging. Magn Reson Med 2005;53:684-691.

29. Larkman DJ, Hajnal JV, Herlihy AH, Coutts GA, Young IR, Ehnholm G. Use of multicoil arrays for separation of signal from multiple slices simultaneously excited. J Magn Reson Imaging 2001;13:313-317.

30. Moeller S, Yacoub E, Olman CA, Auerbach E, Strupp J, Harel N, Uğurbil K. Multiband multislice GE-EPI at 7 tesla, with 16-fold acceleration using partial parallel imaging with application to high spatial and temporal whole-brain fMRI. Magn Reson Med 2010;63:1144-1153.

31. Setsompop K, Gagoski BA, Polimeni JR, Witzel T, Wedeen VJ, Wald LL. Blipped-controlled aliasing in parallel imaging for simultaneous multislice echo planar imaging with reduced g-factor penalty. Magn Reson Med 2012;67:1210-1224. 